\title{
5 x 25 Gbit/s WDM Transmitters Based on Passivated Graphene-Silicon Electro-Absorption Modulators
}

\author{
Chiara Alessandri ${ }^{1,2,}{ }^{*}$, Inge Asselberghs ${ }^{1}$, Steven Brems ${ }^{1}$, Cedric Huyghebaert $^{1}$, Joris \\ Van Campenhout ${ }^{1}$, Dries Van Thourhout ${ }^{2}$, and Marianna Pantouvakı ${ }^{1}$ \\ ${ }^{1}$ Imec, Kapeldreef 75, 3001 Leuven, Belgium \\ ${ }^{2}$ Ghent University-imec, Department of information Technology, Technologiepark-Zwijnaarde 15, 9052 Gent, Belgium \\ ${ }^{*}$ Corresponding author: chiara.alessandri@ugent.be
}

\begin{abstract}
Today, one of the key challenges for the exploitation of graphene devices is establishing fabrication processes that can ensure performance stability, repeatability and that can eventually enable production in high volumes. In this paper we use up-scalable fabrication processes to demonstrate three 5channel wavelength-division multiplexing (WDM) transmitters, each based on 5 graphene-silicon electroabsorption modulators (EAM). A passivation-first approach is used to encapsulate graphene, which results in hysteresis-free and uniform performance across the 5 channels of each WDM transmitter, for a total of 15 modulators. Open eye diagrams are obtained at $25 \mathrm{~Gb} / \mathrm{s}$ using $2.5 \mathrm{~V}_{\mathrm{pp}}$, thus demonstrating potential for multi-channel data transmission at $5 \times 25 \mathrm{~Gb} / \mathrm{s}$ on each of the three WDM transmitters.
\end{abstract}

\section{INTRODUCTION}

As cloud computing, big data applications and social networking are expected to keep growing exponentially, the amount of annual global data center traffic is set to surpass 20 ZB by 2021 [1]. To meet this demand, it's estimated that data center operators will have to upgrade their network to $1.6 \mathrm{~Tb} / \mathrm{s}$ by 2022 [2]. Advanced multiplexing technologies represent an effective solution to achieve a network infrastructure that can carry more data more efficiently. [3, 4] Wavelength-division multiplexing (WDM) uses different channels to carry signals at different wavelengths in a single optical fiber or waveguide simultaneously. $[5,6]$ Next to WDM, other multiplexing solutions are space-division multiplexing (SDM) [7], based on multi-core waveguides, modedivision multiplexing (MDM) [8], with multiple guided modes, and polarisation-division multiplexing (PDM), using two orthogonal polarisations together. These approaches have independent degrees of freedom and can be combined to form hybrid multiplexing systems with capacity up to Pbit/s [4]. Among these, wavelength-division multiplexing (WDM) links, enabled by low loss, broadband and low power consumption modulators, are one of the most successful technology. WDM allows to exploit the full bandwidth of existing optical fibers, leading to a reduced construction cost. In addition, it is simple to implement as channels can be flexibly added or removed, and the active optical equipment is shared by the different channels.

Graphene has attracted interest in recent years due to its characteristic broadband absorption, that ranges from visible to infrared. Graphene's absorption can be easily tuned through capacitive charging by applying an electric field [9], and has therefore the potential to enable active optoelectronic functionality
Table 1. Waveguide width $\left(\mathrm{W}_{\mathrm{wg}}\right)$ and device length $\left(\mathrm{L}_{\text {device }}\right)$ values used to fabricate the three WDM transmitters. Increasing $W_{w g}$ and $L_{\text {device }}$ is expected to increase the extinction ratio but also the device capacitance, and therefore to reduce the $3 \mathrm{~dB}$ bandwidth.

\begin{tabular}{ccc}
\hline & $\mathbf{W}_{\mathbf{w g}}(\mathbf{n m})$ & $\mathbf{L}_{\text {device }}(\boldsymbol{\mu m})$ \\
\hline WDM1 & 500 & 100 \\
WDM2 & 600 & 100 \\
WDM3 & 600 & 150 \\
\hline
\end{tabular}

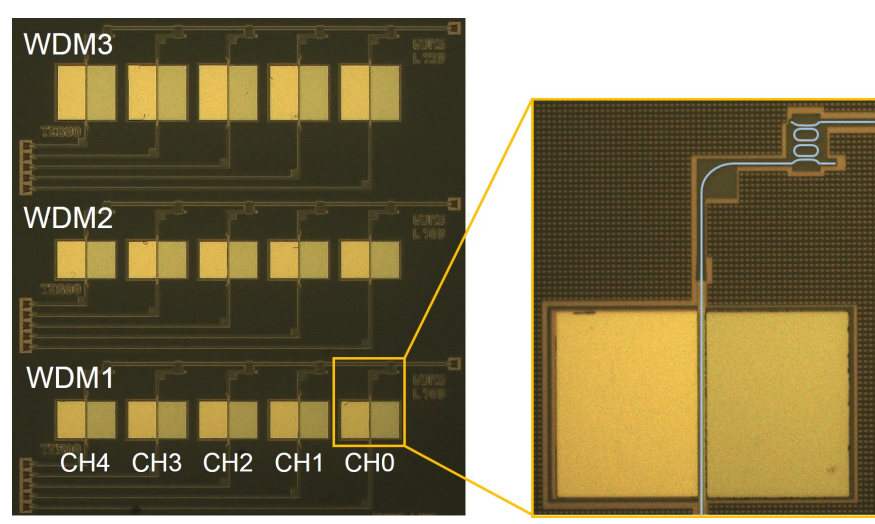

Fig. 1. Top view microscope image showing the three WDM transmitters, each based on 5 graphene-Si EAMs and 5 second order MRRs. 
onto passive optical waveguides, such as $\mathrm{Si}$ or $\mathrm{SiN}$ waveguides $[10,11]$. These properties, together with high carrier mobility, make graphene an attractive material for high-speed photonic devices [12, 13], such as modulators [10, 14-21] and photodetectors [22-25]. Graphene-based modulators can therefore be implemented in WDM systems to modulate the signal on the different channels. Graphene integration in photonics has already been demonstrated for Mach-Zender modulators (MZM) [18], microring modulators (MRM) [10] and electro-absorption modulators (EAM) [14-17, 19, 21]. Compared to graphene MZMs and MRMs, graphene EAMs offer some advantages. Graphene MZMs have wide optical bandwidth and high extinction ratio (ER), but suffer from high insertion loss (IL), high power consumption and large device footprint [18]. On the other hand, graphene MRMs offer lower power consumption and smaller device footprint, but fail to exploit graphene's characteristic broadband absorption due to the resonant nature of the microring [10]. In addition, MRMs suffer from tight fabrication tolerance, therefore thermal stabilisation is necessary to match the resonant wavelength of the ring to the incoming wavelength. Graphene-based EAMs offer very wide optical bandwidth (up to $180 \mathrm{~nm}$ in the C-band) [17], potentially low power consumption, and low IL $[15,16]$.

So far, the demonstration of graphene modulators has been focused on individual components, due to challenges in processing, transfer and integration of high quality graphene at large scale [26]. In this work, we demonstrate for the first time the integration of multiple graphene EAMs with functional silicon photonics circuits. We use three 5-channel WDM transmitters, each based on 5 graphene-Si EAMs designed for TE-polarised light (see Table 1) and Si-based second-order microring resonators (MRR) for wavelength multiplexing, to demonstrate uniform and hysteresis-free performance across 15 graphene EAMs. To achieve this, we employ up-scalable fabrication processes and a passivation-first approach to encapsulate the graphene layer. The three WDM transmitters are fabricated varying the waveguide width and the device length of the EAMs. For the first and second WDM transmitters, we report $5.5 \mathrm{~dB}$ and $5.6 \mathrm{~dB}$ ER across $10 \mathrm{~nm}$ bandwidth for 5 identical $100 \mu \mathrm{m}$-long devices with $500 \mathrm{~nm}$ - and $600 \mathrm{~nm}$-wide waveguides respectively. On the third transmitter, with $150 \mu \mathrm{m}$-long EAMs, we achieve $8.1 \mathrm{~dB}$ ER. Open eye diagrams are measured at $25 \mathrm{~Gb} / \mathrm{s}$ using $2.5 \mathrm{~V}_{\mathrm{pp}}$ on each of the five channels of the three WDM transmitters, thus demonstrating potential for data transmission at $5 \times 25 \mathrm{~Gb} / \mathrm{s}$. Compared with our preliminary results presented in [27] we show an extra channel and more detailed device characterisation.

\section{DESIGN AND FABRICATION}

We fabricated three WDM transmitters consisting of 5 graphene EAMs and 5 second order MRRs each, as shown in Fig.1. Each transmitted wavelength goes through the graphene EAMs before being added to the bus waveguide of the MRRs. The channel spacing of the MRRs, acting as multiplexer (MUX), is designed to fit a grid spacing of $300 \mathrm{GHz}(2.4 \mathrm{~nm})$ and a free-spectral range (FSR) of $12 \mathrm{~nm}$. The rings have a racetrack shape and are implemented with $450 \mathrm{~nm}$-wide waveguides, $9 \mu \mathrm{m}$ coupling length, 5 $\mu \mathrm{m}$ radius and $190 \mathrm{~nm}$ bus-ring gap [5]. To reduce fabrication complexity and power consumption, no temperature control was used, therefore variations in IL, resonant wavelength and cross-talk are expected due to local non-uniformities, as shown in [5]. The first transmitter (WDM1) is made of graphene EAMs

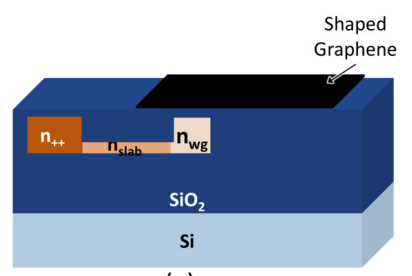

(a)

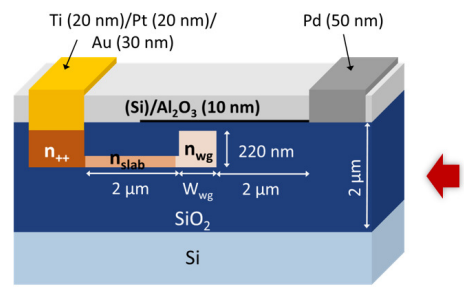

(d)

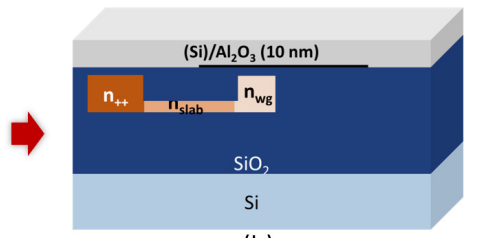

(b)

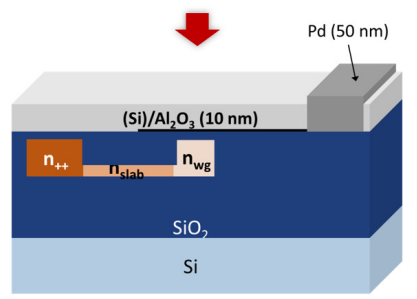

(c)
Fig. 2. Main steps of the process flow used to fabricate the graphene-Si EAMs: (a) graphene shaping, (b) Si $(0.5 \mathrm{~nm}) / \mathrm{Al}_{2} \mathrm{O}_{3}(10 \mathrm{~nm})$ deposition, (c) graphene contact, $(\mathrm{d})$ Si contact. The passivation layer on graphene helps obtaining uniform and hysteresis-free performance. The Si waveguide is connected to a TE-mode fiber grating coupler.

with $500 \mathrm{~nm}$-wide waveguides and $100 \mu \mathrm{m}$-long graphene. The second (WDM2) and third (WDM3) transmitters are made of graphene EAMs with $600 \mathrm{~nm}$-wide waveguides and $100 \mu \mathrm{m}$ and $150 \mu \mathrm{m}$-long graphene respectively. The MRRs are connected to the EAMs using tapers. Increasing the waveguide width $\left(\mathrm{W}_{\mathrm{wg}}\right)$ and the device length $\left(\mathrm{L}_{\text {device }}\right)$ is expected to increase the extinction ratio but also the device capacitance, and therefore to reduce the $3 \mathrm{~dB}$ bandwidth. Each graphene EAM is based on a $220 \mathrm{~nm}$-thick n-doped Si waveguide, fabricated on a SOI wafer with $2 \mu \mathrm{m}$ buried oxide in imec's $200 \mathrm{~mm}$ Si photonics platform. The waveguide is partially etched on one side, leaving a $70 \mathrm{~nm}$ slab for electrical contact to $\mathrm{Si}$. It is embedded in $\mathrm{SiO}_{2}$ to ensure a planar surface for the subsequent graphene transfer and is designed to transmit TE-polarised light in the C-band (Fig. 2). Three separate doping levels are used to minimise the Si contact and sheet resistance, without significantly increasing the waveguide loss.

After oxide chemical-mechanical planarisation (CMP), the processing is continued at coupon level in a lab environment and graphene grown by chemical vapour deposition (CVD), commercially available from Graphenea, is transferred onto the substrate. Graphene-based devices show high sensitivity to environmental factors, such as ambient air, organic solvents and lithography resists used for fabrication [28-31]. Adsorbates on the graphene surface cause unintentional doping, while adsorbates at the graphene $/ \mathrm{SiO}_{2}$ interface affect the performance of graphene devices by introducing trap states that cause hysteretic behaviour [32]. The stability of graphene devices can be improved by encapsulating graphene with a protective layer. A high- $\kappa$ dielectric material is expected to screen the charged impurities at the graphene $/ \mathrm{SiO}_{2}$ interface and, if deposited at the beginning of the fabrication flow (passivation-first approach), it also protects graphene from contacting organic solvents and lithography resists during processing. Therefore, the device fabrication is carried out following a passivation-first approach. First, graphene is patterned to cover part of the Si waveguide and to define the length of the EAM (Fig. 2a). After patterning, 


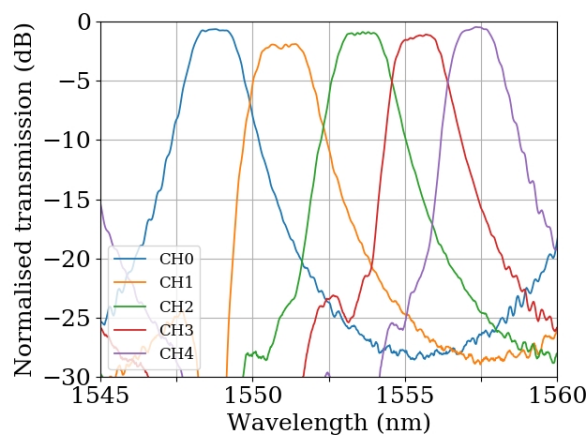

(a)

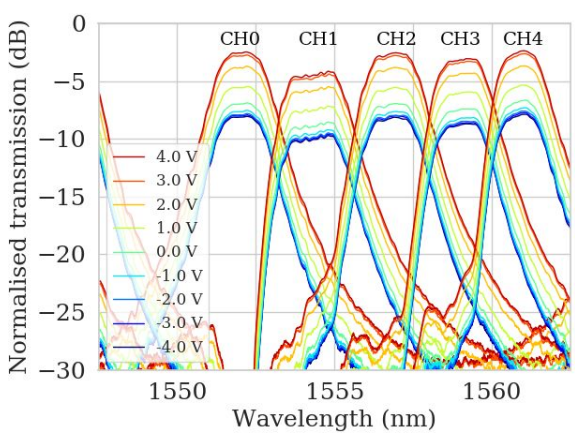

(b)

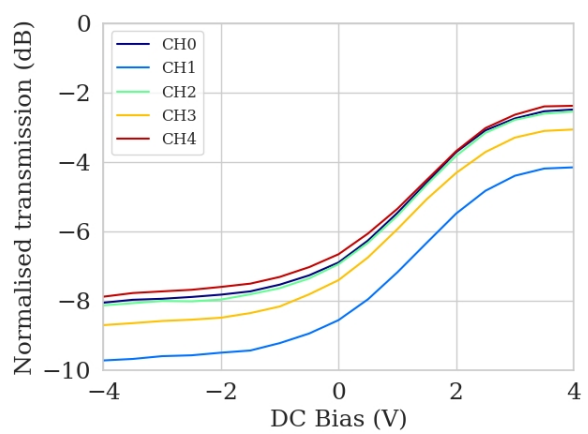

(c)

Fig. 3. (a) Transmission spectra, normalised to a reference waveguide, measured on a WDM filter without graphene, showing the insertion loss of the second order MRRs. (b) Transmission spectra on WDM2, normalised to a reference waveguide without graphene. The voltage is varied from $-4 \mathrm{~V}$ to $4 \mathrm{~V}$ on each graphene EAM, resulting in the tuning of the transmission on each channel. (c) Normalised transmission as a function of DC voltage bias, measured on WDM2. The transmission is extracted at the peak wavelength of each channel. The modulation is uniform on the 5 channels.
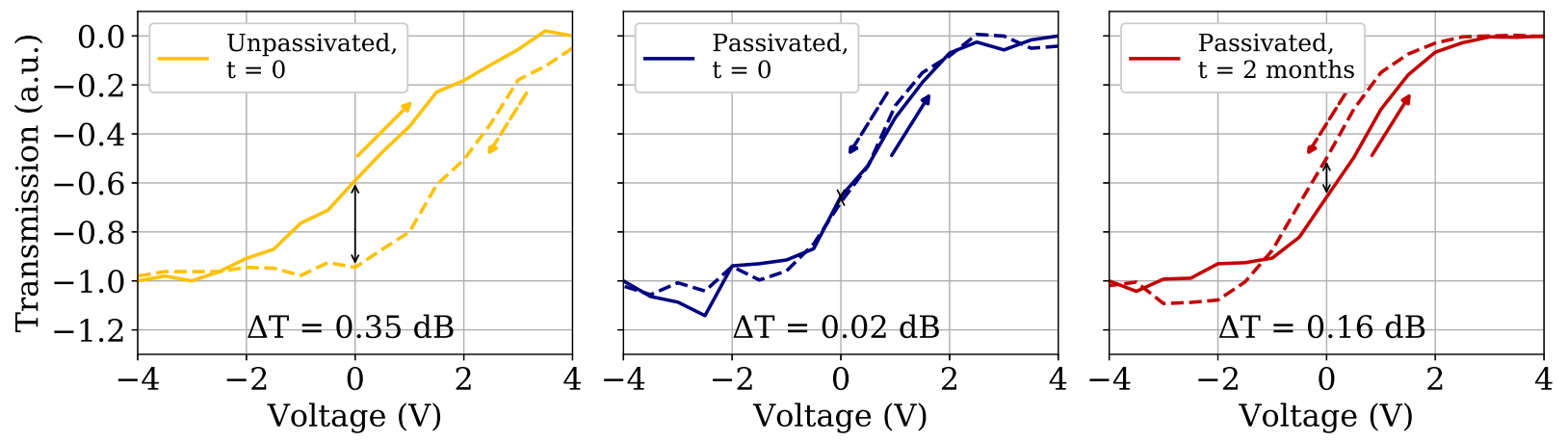

Fig. 4. Comparison of transmission curves, measured with a double voltage sweep right after fabrication, between an unpassivated (yellow) and a passivated (blue) $25 \mu \mathrm{m}$-long graphene EAMs. The unpassivated EAM shows hysteretic behaviour, which is not present on the passivated EAM. The same measurement is repeated on the passivated EAM after 2 months time (red), showing a small hysteresis that remains less pronounced compared to the unpassivated device.

graphene is passivated with an $\mathrm{Al}_{2} \mathrm{O}_{3}$ layer (Fig. 2b). Depositing the dielectric after, and not before, patterning allows to obtain full sample coverage and reduce intercalation of solvents between graphene and $\mathrm{SiO}_{2}$ during processing. $\mathrm{Al}_{2} \mathrm{O}_{3}$ is chosen as encapsulating material because it allows to obtain hysteresis-free electro-optical response, it preserves the p-doping characteristic of unpassivated graphene and it's stable over time [33]. To ensure a uniform passivation layer, we first evaporate $0.5 \mathrm{~nm}$ of $\mathrm{Si}$ as seeding layer by e-gun evaporation, and then deposit $10 \mathrm{~nm}$ of $\mathrm{Al}_{2} \mathrm{O}_{3}$ as capping layer by atomic layer deposition (ALD) [33]. Contacts are made to graphene $(50 \mathrm{~nm} \mathrm{Pd})$ and to the doped Si $(20 \mathrm{~nm} \mathrm{Ti} / 20 \mathrm{~nm} \mathrm{Pt} / 30 \mathrm{~nm} \mathrm{Au})$ using a lift-off process (Fig. 2c and Fig. 2d). Due to the presence of the passivation layer, an etching step has to be added to remove the $\mathrm{Al}_{2} \mathrm{O}_{3}$ before depositing Pd to contact graphene. As a consequence, graphene is removed from the contact area, and a side contact between graphene and Pd is created. This contacting scheme has been shown to reduce the metal-graphene contact resistance compared to a standard top contact [34]. The contacts are placed $2 \mu \mathrm{m}$ away from the waveguide and therefore have no impact on transmission loss. Graphene and the Si waveguide are separated by a $\mathrm{SiO}_{2}$ layer of $5 \mathrm{~nm}$, thus forming a Graphene-Oxide-Si (GOS) capacitor (Fig. 2d). The metal contacts are used to apply
Table 2. Insertion loss (IL) and extinction ratio (ER) at $8 \mathrm{~V}_{\mathrm{pp}}$. The values are averaged over 5 channels. WDM3 exhibits higher IL and ER due to the longer $\mathrm{L}_{\text {device}}$.

\begin{tabular}{cccc}
\hline & $\mathbf{L}_{\text {device }}(\boldsymbol{\mu m})$ & IL $(\mathbf{d B})$ & ER (dB) \\
\hline WDM1 & 100 & $3.8 \pm 1.0$ & $5.5 \pm 0.1$ \\
WDM2 & 100 & $2.9 \pm 0.7$ & $5.6 \pm 0.1$ \\
WDM3 & 150 & $4.0 \pm 0.5$ & $8.1 \pm 0.7$ \\
\hline
\end{tabular}

an electric field across the GOS capacitor. Due to the electric field, charges are accumulated or depleted in the graphene layer, and consequently the graphene absorption is tuned as a function of the applied voltage bias [9].

\section{RESULTS AND DISCUSSION}

We first performed unbiased fiber-to-fiber transmission measurements of the three WDM transmitters, each composed of 5 channels. The insertion loss (IL) of each channel was calculated as the peak transmission of the channel, normalised to the transmission of a reference waveguide without graphene at the 
Table 3. Total resistance $\left(\mathrm{R}_{\mathrm{tot}}\right)$ and $\mathrm{GOS}$ capacitance $\left(\mathrm{C}_{\mathrm{GOS}}\right)$ extracted from $S_{11}$ parameter fitting, simulated (from the fitted parameters in column 1 and 2) and measured $f_{3 \mathrm{~dB}}$ at $0 \mathrm{~V}$. The values are averaged over 5 channels. Due to the longer $L_{\text {device, }}$ WDM3 exhibits higher RC constant, and therefore lower $\mathrm{f}_{3 \mathrm{~dB}}$.

\begin{tabular}{lccccc}
\hline & \multirow{2}{*}{$\mathbf{L}_{\text {device }}(\boldsymbol{\mu m})$} & $\mathbf{R}_{\text {tot }}(\Omega)$ & $\mathbf{C}_{\mathrm{GOS}}(\mathbf{f F})$ & \multicolumn{2}{c}{$\mathbf{f}_{3 \mathrm{~dB}}(\mathrm{GHz})$ at 0 V } \\
& & & & Simulated & Measured \\
\hline WDM1 & 100 & $78 \pm 5$ & $112.6 \pm 0.5$ & $10.1 \pm 0.5$ & $9.5 \pm 0.7$ \\
WDM2 & 100 & $65 \pm 5$ & $134.7 \pm 0.5$ & $9.5 \pm 0.5$ & $9.3 \pm 0.1$ \\
WDM3 & 150 & $49 \pm 5$ & $206.6 \pm 0.5$ & $7.0 \pm 0.5$ & $7.1 \pm 0.3$ \\
\hline
\end{tabular}

same wavelength. The extracted average and standard deviation values of IL over the 5 channels are $3.8 \pm 1.0 \mathrm{~dB}, 2.9 \pm 0.7 \mathrm{~dB}$ and $4.0 \pm 0.5 \mathrm{~dB}$ for WDM1, WDM2 and WDM3 respectively (Table 2). To determine the main source of insertion loss, we performed transmission measurements on a WDM filter without graphene, with same design as WDM2, located on a different die. These measurements, normalised to a reference waveguide, show that the IL due to the second order MRRs is $\sim 2 \mathrm{~dB}$ for $\mathrm{CH} 1$ and less than $1 \mathrm{~dB}$ for all other channels (Fig. 3a). Therefore, we conclude that the loss of the WDM transmitters is dominated by the IL of the graphene modulators.

The electro-optical response of the graphene EAMs was characterised by sweeping the wavelength from $1510 \mathrm{~nm}$ to 1600 $\mathrm{nm}$ on each channel, while applying voltage bias ranging from $-4 \mathrm{~V}$ to $4 \mathrm{~V}$. The optical power was measured at the output of the transmitters (bus waveguide). An example of this measurement performed on WDM2 is shown in Fig.3b (with reduced wavelength range from $1552.5 \mathrm{~nm}$ to $1562.5 \mathrm{~nm}$ for clarity). The extinction ratio (ER) at $8 \mathrm{~V}_{\mathrm{pp}}$ was obtained by extracting the transmission as a function of the voltage at the peak transmission wavelength of each channel (example for WDM2 in Fig. 3c). The ER is consistent across all the channels, with average values of $5.5 \pm 0.1 \mathrm{~dB}$ for WDM1, $5.6 \pm 0.1 \mathrm{~dB}$ for WDM2 and $8.1 \pm$ $0.7 \mathrm{~dB}$ for WDM3 (Table 2). The higher ER in WDM3 is due to the longer device length, which ensures a longer interaction between the graphene layer and the evanescent field of the light travelling through the waveguide. The electro-optical switching in transmission occurs around $0 \mathrm{~V}$, because of $\mathrm{p}$-doping in graphene. The $\mathrm{p}$-doping characteristic is ideal for electro-optical modulation, because it allows to operate the device at low voltage DC bias. The carrier mobility of graphene is estimated to be $\sim 800 \mathrm{~cm}^{2} /(\mathrm{Vs})$ from measurements performed on electrical test structures fabricated on the same sample. The static power consumption at $-1 \mathrm{~V}$ is calculated to be $<2 \times 10^{-8} \mathrm{~mW}$, due to the $<20$ pA measured leakage current.

In order to study the effect of the $\mathrm{Al}_{2} \mathrm{O}_{3}$ passivation layer on the hysteretic behaviour of the EAMs, we performed double sweep electro-optical measurements at $1560 \mathrm{~nm}$ wavelength on a stand-alone $25 \mu \mathrm{m}$-long graphene EAM fabricated simultaneously on the same chip. We compared these results with the ones obtained on an unpassivated $25 \mu \mathrm{m}$-long graphene EAM fabricated under the same processing conditions (Fig. 4). The unpassivated EAM shows hysteretic behaviour, with a difference in transmission at $0 \mathrm{~V}$ between the forward and backward voltage sweep of $\Delta \mathrm{T}=0.35 \mathrm{~dB}$. This behaviour is not present on the passivated EAM, where $\Delta \mathrm{T}$ is only $0.02 \mathrm{~dB}$, thus showing that the $\mathrm{Al}_{2} \mathrm{O}_{3}$ passivation layer allows to obtain hysteresis-free electro-optical response, while at the same time preserving the p-doping characteristic of unpassivated graphene. The same

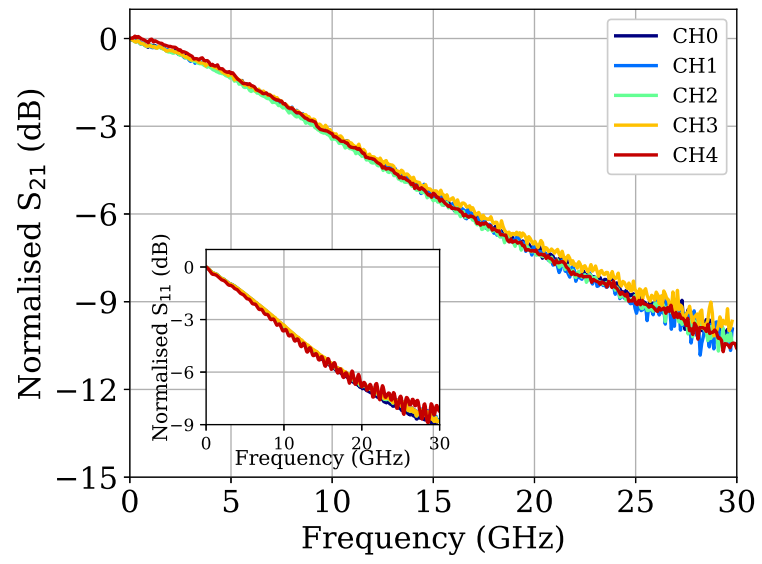

(a)
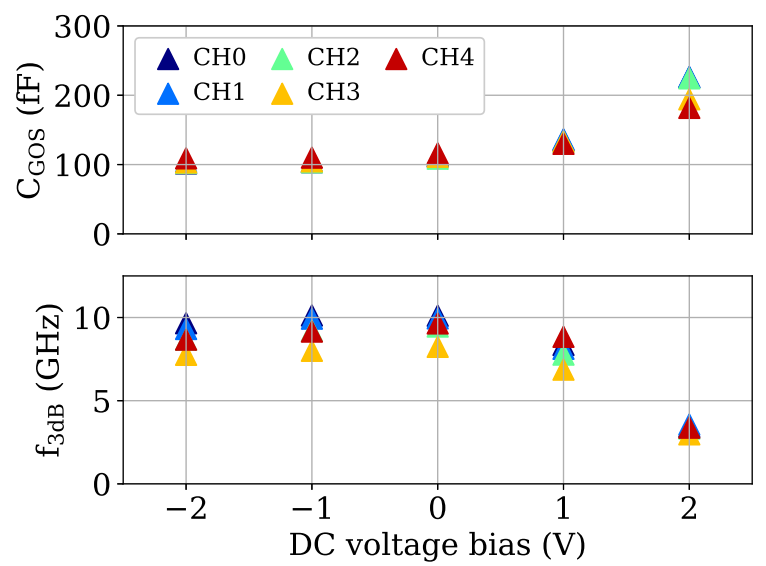

(b)

Fig. 5. (a) Electro-optical $S_{21}$ (inset: $S_{11}$ ) frequency response measured at $0 \mathrm{~V} D C$ bias on WDM2. The response is uniform across the 5 channels. (b) GOS capacitance and $3 \mathrm{~dB}$ bandwidth of WDM1 as a function of DC bias. The GOS capacitance increases at forward bias, causing a drop in $3 \mathrm{~dB}$ bandwidth. The ideal operating region is therefore at $0 \mathrm{~V}$ or low reverse bias.

measurement repeated on the passivated EAM after 2-months time shows no significant degradation in the response of the device $(\Delta T=0.16 \mathrm{~dB})$. Two parameters could be optimised to further improve stability over time. The first is the thickness of the $\mathrm{Al}_{2} \mathrm{O}_{3}$, as it has been shown to affect the performance stability of graphene field-effect devices [33]. In addition, a thick $\mathrm{SiO}_{2}$ layer $(\sim 1 \mu \mathrm{m})$ could be deposited on top of the $\mathrm{Al}_{2} \mathrm{O}_{3}$ to increase further the thickness of the dielectric stack. The second is the type of seeding layer used for $\mathrm{Al}_{2} \mathrm{O}_{3}$ deposition, because it has a significant impact on the dielectric constant $(\kappa)$ and morphology of $\mathrm{ALD}_{2} \mathrm{O}_{3}$ [35]. A higher $\kappa$ would also allow to achieve a better mobility retention in graphene [36], leading to lower graphene resistance and to higher ER.

The electro-optical $S_{21}$ frequency response was measured between $100 \mathrm{MHz}$ and $30 \mathrm{GHz}$ on the three WDM transmitters at DC bias ranging from $-2 \mathrm{~V}$ to $2 \mathrm{~V}$ with a vector network analyser, using $-8 \mathrm{dBm}$ RF power. Fig.5a shows the $S_{21}$ and $\mathrm{S}_{11}$ frequency response of WDM2 at $0 \mathrm{~V} \mathrm{DC}$ bias. The trend of the $3 \mathrm{~dB}$ bandwidth as a function of DC bias is shown in Fig.5b for WDM1. The highest $3 \mathrm{~dB}$ bandwidth is measured at 


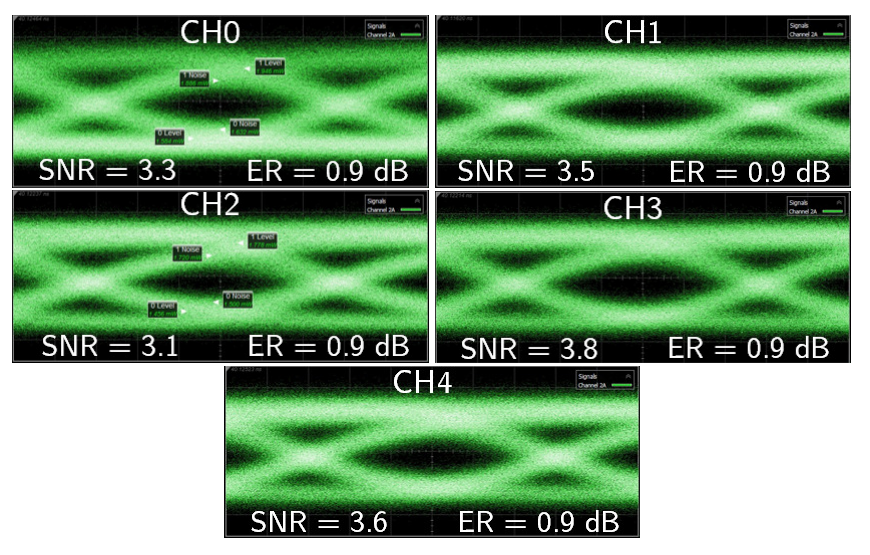

Fig. 6. Eye diagrams measured at $25 \mathrm{Gbit} / \mathrm{s}$ on WDM2 at 2.5 $\mathrm{V}_{\mathrm{pp}}$ and $-1.2 \mathrm{~V}$ DC bias. The performance is uniform across the 5 channels. The dynamic extinction ratio is limited by the low modal overlap with graphene when using TE-polarised light.
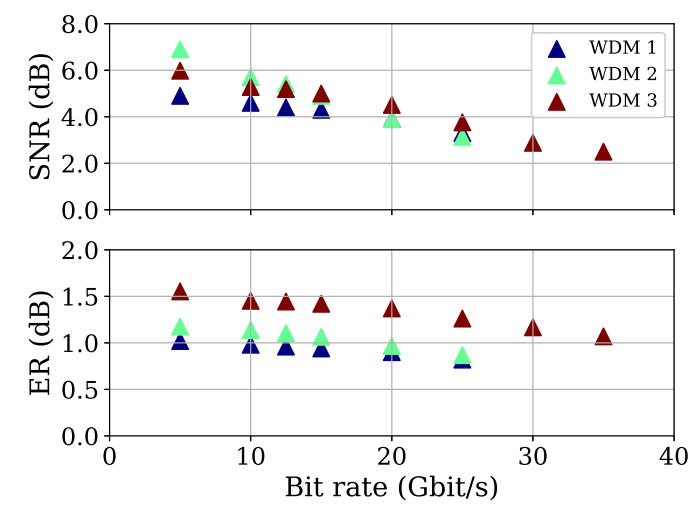

Fig. 7. SNR and dynamic ER as a function of bit rate. The SNR is higher than 3.0 up to $25 \mathrm{Gbit} / \mathrm{s}$ for all the WDM transmitters. The ER for WDM3 is higher than $1 \mathrm{~dB}$ up to $30 \mathrm{Gbit} / \mathrm{s}$, due to the longer device length.

$-1 \mathrm{~V}$ and $0 \mathrm{~V}$, where the total $\mathrm{RC}$ constant of the device reaches the minimum. At reverse bias graphene's neutrality point is approached, therefore the total resistance of graphene increases, and the $3 \mathrm{~dB}$ bandwidth decreases slightly. At forward bias the GOS capacitor with n-doped Si enters the accumulation region, characterised by a drastic increase in the capacitance (Fig. 5b), causing a drop in the $3 \mathrm{~dB}$ bandwidth. Average $3 \mathrm{~dB}$ bandwidths of $9.5 \pm 0.7 \mathrm{GHz}, 9.3 \pm 0.1 \mathrm{GHz}$ and $7.1 \pm 0.3 \mathrm{GHz}$ were recorded respectively for WDM1, WDM2 and WDM3 at $0 \mathrm{~V}$ DC bias. The response decreases as the waveguide width and the graphene length increase, due to the higher RC constant (Table 3).

Eye diagrams were measured at the peak wavelength of each channel using $2^{7}-1$ pseudorandom binary sequence (PRBS) at 2.5 $\mathrm{V}_{\mathrm{pp}}$ with a $50 \Omega$ terminated probe. The applied DC bias is different for each channel because of small variations in graphene doping, with an average value of $-1.2 \pm 0.2 \mathrm{~V}$. Open and symmetrical eye diagrams were generated from $5 \mathrm{~Gb} / \mathrm{s}$ to $25 \mathrm{~Gb} / \mathrm{s}$ for all the channels, thus allowing to transmit data up to $5 \mathrm{x}$ $25 \mathrm{~Gb} / \mathrm{s}$ on each WDM transmitter. Eye diagrams measured on the 5 channels of WDM2 are shown in Fig.6. The dynamic ER and signal-to-noise ratio (SNR) of the 5 channels on each WDM transmitter are reported in Fig.7 as a function of bit rate. The SNR is higher than 3.0 up to $25 \mathrm{Gbit} / \mathrm{s}$ for all the WDM

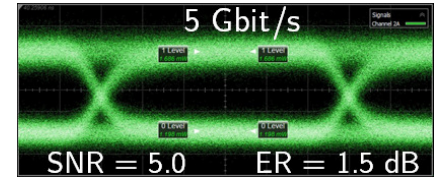

(a)

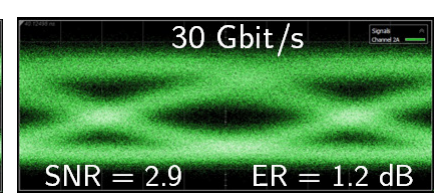

(b)
Fig. 8. Eye diagrams measured at $5 \mathrm{Gbit} / \mathrm{s}$ (a) and $30 \mathrm{Gbit} / \mathrm{s}$ (b) on $\mathrm{CH} 2$ of WDM3 at $2.5 \mathrm{~V}_{\mathrm{pp}}$ and $-1.2 \mathrm{~V}$ DC bias.

transmitters. WDM3 exhibits a $45 \%$ higher ER, due to the longer graphene waveguide coverage, thus allowing to obtain open eye diagrams up to $30 \mathrm{~Gb} / \mathrm{s}$ with an SNR of 2.9 and a dynamic ER of $1.2 \mathrm{~dB}$ (Fig.8). This shows that the primary limiting factor of these devices is the extinction ratio, followed by the frequency response. The dynamic energy consumption $\left(E_{b i t}=C V^{2} / 4\right)$ of a single graphene EAM at $-1 \mathrm{~V}$ is estimated to be $\sim 163 \mathrm{fJ}$ for WDM1, 195 fJ for WDM2 and $\sim 308 \mathrm{fJ}$ for WDM3. These values are, to the best of our knowledge, the lowest reported for graphene-based modulators.

To further improve the performance of these devices, the thickness of the oxide between graphene and $\mathrm{Si}$ can be increased to reduce the device capacitance and therefore the RC constant. However, this leads to a lower modulation efficiency, due to the increased DC bias and $V_{p p}$ necessary to operate the device. Therefore, we suggest a different approach. The Si doping in the waveguide can be optimised to reduce the Si capacitance and resistance contributions in the operating region. For example, an improvement of $\sim 42 \%$ in $3 \mathrm{~dB}$ frequency response can be achieved by $\mathrm{p}$-doping instead of $\mathrm{n}$-doping the Si waveguide without affecting the modulation efficiency, because the device can be operated in depletion mode instead of accumulation mode [37]. A two-fold improvement in ER can be attained by designing graphene-based WDM transmitters for TM- instead of TE-polarised light [15]. Furthermore, an improvement of the graphene quality, and therefore of the carrier mobility of graphene, will allow to increase the ER for fixed $V_{p p}$, reduce the graphene resistance, and reduce the IL of the graphene EAMs.

\section{CONCLUSION}

We demonstrated three graphene-based 5-channel WDM transmitters, fabricated with an up-scalable fabrication process and with passivated graphene, to ensure uniform and hysteresis-free device performance. On each channel, the TE-polarised light is modulated using broadband graphene EAMs, for a total of 15 working devices. On the first and second transmitters, with 100 $\mu \mathrm{m}$-long graphene EAMs, we achieved average static extinction ratios of $5.5 \pm 0.1 \mathrm{~dB}$ and $5.6 \pm 0.1 \mathrm{~dB}$ respectively. On the third transmitter, with $150 \mu \mathrm{m}$-long graphene EAMs, we achieved 8.1 $\pm 0.7 \mathrm{~dB}$ average static extinction ratio. Open eye diagrams were measured in the C-band at $2.5 \mathrm{~V}_{\mathrm{pp}}$ on three WDM transmitters, thus demonstrating potential for data transmission at $5 \times 25$ $\mathrm{Gb} / \mathrm{s}$.

\section{FUNDING}

Horizon 2020 Framework Programme (785219).

\section{ACKNOWLEDGMENTS}

The authors acknowledge imec's industrial affiliation Optical I/O program. The authors acknowledge Ashwyn Srinivasan 
and Peter De Heyn for useful discussions.

\section{DISCLOSURES}

The authors declare no conflicts of interest.

\section{REFERENCES}

1. "Cisco Global Cloud Index: Forecast and Methodology, 2016-2021," https://www.cisco.com/c/en/us/solutions/collateral/service-provider/ global-cloud-index-gci/white-paper-c11-738085.html (2018).

2. "2019 Ethernet Alliance Roadmap," http://www.ethernetalliance.com (2019).

3. P. J. Winzer, "Making spatial multiplexing a reality," Nat. Photonics 8, 345-348 (2014).

4. D. Dai and J. E. Bowers, "Silicon-based on-chip multiplexing technologies and devices for Peta-bit optical interconnects," Nanophotonics 3, 283-311 (2014).

5. P. De Heyn, J. De Coster, P. Verheyen, G. Lepage, M. Pantouvaki, P. Absil, W. Bogaerts, J. Van Campenhout, and D. Van Thourhout, "Fabrication-tolerant four-channel wavelength-division-multiplexing filter based on collectively tuned si microrings," J. Light. Technol. 31, 27852792 (2013).

6. J. B. Driscoll, P. Doussiere, S. Islam, R. Narayan, W. Lin, H. Mahalingam, J. S. Park, Y. Lin, K. Nguyen, K. Roelofs, A. Dahal, R. Venables, L. Liao, R. Jones, D. Zhu, S. Priyadarshi, B. Parthasarathy, and Y. Akulova, "First 400G 8-Channel CWDM Silicon Photonic Integrated Transmitter," IEEE Int. Conf. on Group IV Photonics (GFP) (2018).

7. K. Igarashi, D. Soma, Y. Wakayama, K. Takeshima, Y. Kawaguchi, N. Yoshikane, T. Tsuritani, I. Morita, and M. Suzuki, "Ultra-dense spatialdivision-multiplexed optical fiber transmission over 6-mode 19-core fibers," Opt. Express 24, 10213 (2016).

8. D. Zhou, C. Sun, Y. Lai, Y. Yu, and X. Zhang, "Integrated silicon multifunctional mode-division multiplexing system," Opt. Express 27, 10798 (2019).

9. G. W. Hanson, "Dyadic Green's functions and guided surface waves for a surface conductivity model of graphene," J. Appl. Phys. 103 (2008).

10. C. T. Phare, Y.-H. Daniel Lee, J. Cardenas, and M. Lipson, "Graphene electro-optic modulator with $30 \mathrm{GHz}$ bandwidth," Nat. Photonics 9 , 511-514 (2015).

11. L. Abdollahi Shiramin and D. Van Thourhout, "Graphene Modulators and Switches Integrated on Silicon and Silicon Nitride Waveguide," IEEE J. on Sel. Top. Quantum Electron. 23 (2017).

12. F. Bonaccorso, Z. Sun, T. Hasan, and A. C. Ferrari, "Graphene Photonics and Optoelectronics," Nat. Photonics 4, 611-622 (2010).

13. M. Romagnoli, V. Sorianello, M. Midrio, F. H. L. Koppens, C. Huyghebaert, D. Neumaier, P. Galli, W. Templ, A. D'Errico, and A. C. Ferrari, "Graphene-based integrated photonics for next-generation datacom and telecom," Nat. Rev. Mater. 3, 392-414 (2018).

14. C. Alessandri, I. Asselberghs, Y. Ban, S. Brems, C. Huyghebaert, J. Van Campenhout, D. Van Thourhout, and M. Pantouvaki, "Broadband 20 Gbit/s Graphene-Si Electro-Absorption Modulator," in ECOC, (2018).

15. Y. Hu, M. Pantouvaki, J. Van Campenhout, S. Brems, I. Asselberghs, C. Huyghebaert, P. Absil, and D. Van Thourhout, "Broadband $10 \mathrm{~Gb} / \mathrm{s}$ operation of graphene electro-absorption modulator on silicon," Laser Photonics Rev. 10, 307-316 (2016).

16. M. Liu, X. Yin, E. Ulin-avila, B. Geng, T. Zentgraf, L. Ju, F. Wang, and $X$. Zhang, "A graphene-based broadband optical modulator," Nat. (UK) 474, 64-6767 (2011).

17. M. Mohsin, D. Schall, M. Otto, A. Noculak, D. Neumaier, and H. Kurz, "Graphene based low insertion loss electro-absorption modulator on SOI waveguide," Opt. Express 22, 15292 (2014).

18. V. Sorianello, M. Midrio, G. Contestabile, I. Asselberghs, J. Van Campenhout, C. Huyghebaert, I. Goykhman, A. K. Ott, A. C. Ferrari, and M. Romagnoli, "Graphene-silicon phase modulators with gigahertz bandwidth," Nat. Photonics 12, 40-44 (2018).

19. V. Sorianello, G. Contestabile, M. Midrio, M. Pantouvaki, I. Asselberghs, J. Van Campenhout, C. Huyghebaerts, A. D'Errico, P. Galli, and M. Ro- magnoli, "Chirp management in silicon-graphene electro absorption modulators," Opt. Express 25, 19371 (2017).

20. H. Dalir, Y. Xia, Y. Wang, and X. Zhang, "Athermal Broadband Graphene Optical Modulator with $35 \mathrm{GHz}$ Speed," ACS Photonics 3, 1564-1568 (2016).

21. M. A. Giambra, V. Sorianello, V. Miseikis, S. Marconi, A. Montanaro, P. Galli, S. Pezzini, C. Coletti, and M. Romagnoli, "High-speed double layer graphene electro-absorption modulator on SOI waveguide," Opt. Express 27, 20145 (2019).

22. A. Pospischil, M. Humer, M. M. Furchi, D. Bachmann, R. Guider, T. Fromherz, and T. Mueller, "CMOS-compatible graphene photodetector covering all optical communication bands," Nat. Photonics 7 , 892-896 (2013).

23. X. Gan, R.-J. Shiue, Y. Gao, I. Meric, T. F. Heinz, K. Shepard, J. Hone, S. Assefa, and D. Englund, "Chip-integrated ultrafast graphene photodetector with high responsivity," Nat. Photonics 7, 883-887 (2013).

24. I. Goykhman, U. Sassi, B. Desiatov, N. Mazurski, S. Milana, D. De Fazio, A. Eiden, J. Khurgin, J. Shappir, U. Levy, and A. C. Ferrari, "OnChip Integrated, Silicon-Graphene Plasmonic Schottky Photodetector with High Responsivity and Avalanche Photogain," Nano Lett. 16, 30053013 (2016).

25. D. Schall, C. Porschatis, M. Otto, and D. Neumaier, "Graphene photodetectors with a bandwidth $>76 \mathrm{GHz}$ fabricated in a 6" wafer process line," J. Phys. D: Appl. Phys. 50, 124004 (2017).

26. S. Brems, K. Verguts, N. Vrancken, B. Vermeulen, C. Porret, L. Peters, C. H. Wu, C. Huyghebaert, K. Schouteden, C. Van Haesendonck, and S. De Gendt, "Graphene Synthesis and Transfer Improvements for Applications in the Semiconductor Industry," ECS Transactions 77, 3-13 (2017)

27. C. Alessandri, I. Asselberghs, P. D. Heyn, S. Brems, J. Van Campenhout, and D. Van Thourhout, "4-Channel C-Band WDM Transmitter Based on $10 \mathrm{GHz}$ Graphene-Silicon Electro-Absorption Modulators," in Optical Fiber Communication Conference, (2019).

28. S. Adam, E. H. Hwang, V. M. Galitski, and S. Das Sarma, "A selfconsistent theory for graphene transport," Proc. Natl. Acad. Sci. United States Am. 104, 18392-18397 (2007).

29. H. Wang, Y. Wu, C. Cong, J. Shang, and T. Yu, "Hysteresis of electronic transport in graphene transistors," ACS Nano 4, 13 (2010).

30. P. Joshi, H. E. Romero, A. T. Neal, V. K. Toutam, and S. A. Tadigadapa, "Intrinsic doping and gate hysteresis in graphene field effect devices fabricated on $\mathrm{SiO} 2$ substrates," J. Phys. Condens. Matter 22 (2010).

31. Y. G. Lee, C. G. Kang, U. J. Jung, J. J. Kim, H. J. Hwang, H. J. Chung, S. Seo, R. Choi, and B. H. Lee, "Fast transient charging at the graphene/ SiO2interface causing hysteretic device characteristics," Appl. Phys. Lett. 98, 183508 (2011).

32. H. Xu, Y. Chen, J. Zhang, and H. Zhang, "Investigating the mechanism of hysteresis effect in graphene electrical field device fabricated on $\mathrm{SiO} 2$ substrates using raman spectroscopy," Small. 8, 2833-2840 (2012).

33. A. A. Sagade, D. Neumaier, D. Schall, M. Otto, A. Pesquera, A. Centeno, A. Z. Elorza, and H. Kurz, "Highly air stable passivation of graphene based field effect devices," Nanoscale. 7, 3558-3564 (2015).

34. D. W. Yue, C. H. Ra, X. C. Liu, D. Y. Lee, and W. J. Yoo, "Edge contacts of graphene formed by using a controlled plasma treatment," Nanoscale. 7, 825-831 (2015).

35. B. Fallahazad, K. Lee, G. Lian, S. Kim, C. M. Corbet, D. A. Ferrer, L. Colombo, and E. Tutuc, "Scaling of Al2O3dielectric for graphene field-effect transistors," Appl. Phys. Lett. 100, 10-14 (2012).

36. S. A. Imam, A. Guermoune, M. Siaj, and T. Szkopek, "Oxide and nitride encapsulation of large-area graphene field effect devices," Thin Solid Films 520, 7041-7043 (2012).

37. C. Alessandri, I. Asselberghs, S. Brems, C. Huyghebaert, J. Van Campenhout, D. Van Thourhout, and M. Pantouvaki, "High speed single-layer graphene-Si electro-absorption modulator," in CLEO-PR, (2018). 